\title{
Robustness of superconductivity to competing magnetic phases in tetragonal FeS
}

\author{
Franziska K. K. Kirschner, ${ }^{1,}{ }^{*}$ Franz Lang, ${ }^{1}$ Craig V. Topping, ${ }^{1}$ Peter J. Baker, ${ }^{2}$ Francis L. Pratt, ${ }^{2}$ Sophie E. Wright,${ }^{3}$ \\ Daniel N. Woodruff, ${ }^{3}$ Simon J. Clarke, ${ }^{3}$ and Stephen J. Blundell ${ }^{1, \dagger}$ \\ ${ }^{1}$ Department of Physics, University of Oxford, Clarendon Laboratory, Parks Road, Oxford OX1 3PU, United Kingdom \\ ${ }^{2}$ ISIS Pulsed Neutron and Muon Facility, Rutherford Appleton Laboratory, Chilton, Oxfordshire OX11 OQX, United Kingdom \\ ${ }^{3}$ Department of Chemistry, University of Oxford, Inorganic Chemistry Laboratory, South Parks Road, Oxford OX1 3QR, United Kingdom
}

(Received 4 July 2016; revised manuscript received 8 September 2016; published 12 October 2016)

\begin{abstract}
We have determined the superconducting and magnetic properties of a hydrothermally synthesized powder sample of tetragonal FeS using muon spin rotation ( $\mu \mathrm{SR})$. The superconducting properties are entirely consistent with those of a recently published study, showing fully gapped behavior and giving a penetration depth of $\lambda_{a b}=204(3) \mathrm{nm}$. However, our zero-field $\mu$ SR data are rather different and indicate the presence of a small, nonsuperconducting magnetic phase within the sample. These results highlight that sample-to-sample variations in magnetism can arise in hydrothermally prepared phases, but interestingly the superconducting behavior is remarkably insensitive to these variations.
\end{abstract}

DOI: 10.1103/PhysRevB.94.134509

\section{INTRODUCTION}

Since the discovery of the first iron-based superconductors $[1,2]$, this family of compounds has been a topic of intense interest. Initially, the focus was on iron arsenides for which the superconducting critical temperature $T_{\mathrm{c}}$ could reach $55 \mathrm{~K}$ [3]. Subsequently, it became possible to substantially enhance the superconducting properties of FeSe [4] $\left(T_{\mathrm{c}}=8 \mathrm{~K}\right)$ using pressure [5], molecular intercalation [6], and even thin-film fabrication [7]. Sulfides have received particular attention following the discovery of the first iron-sulfide superconductor, $\mathrm{BaFe}_{2} S_{3}$, reaching a $T_{\mathrm{c}}$ of $14 \mathrm{~K}$ [8]. The record for the highest $T_{\mathrm{c}}$ of any superconductor is currently held by a sulfide (203 K for $\mathrm{H}_{3} \mathrm{~S}$ at high pressure [9]). Until recently, the possibility of studying superconductivity in the simplest sulfide analog of iron selenide, FeS, had not been explored. This layered polymorph, mackinawite, is not trivial to synthesize and had not previously been reported to be a superconductor. Recently, Lai et al. reported a facile hydrothermal synthesis of this polymorph which also produced superconducting samples with a $T_{\mathrm{c}}$ of $\approx 5 \mathrm{~K}$ [10]. Pachmayr et al. used single-crystal $\mathrm{x}$-ray diffraction measurements to show that such samples are stoichiometric FeS [11].

A variety of ground states have been predicted for $t$-FeS, including nonmagnetic metallic [12-14] and stripe antiferromagnetic order [15]. It is thought that the metallic state may arise due to delocalized iron $d$ electrons [16]. It has been found that both the superconducting and magnetic properties of $t$-FeS are strongly dependent on fine details in the crystal structure [17]. Density functional theory calculations have shown that $t$-FeS is close to a spin-density wave (SDW) instability and that the electronic structure and Fermi surface are very close to those of $\mathrm{FeSe}[12,14]$. The $\mathrm{FeS}_{4}$ tetrahedra in $t$-FeS are closer to being perfectly regular [10] than those in FeSe [18] (the S-Fe-S angle is 110.8(2) compared to $104.02^{\circ}$ for $\mathrm{Se}-\mathrm{Fe}-\mathrm{Se}$ ), and even though this normally favors superconductivity in arsenides [19], the value of $T_{\mathrm{c}}$

\footnotetext{
*franziska.kirschner@physics.ox.ac.uk

${ }^{\dagger}$ stephen.blundell@physics.ox.ac.uk
}

in $t$-FeS is lower than that in FeSe. It has been suggested that a low-moment magnetic phase with $T_{\mathrm{N}} \approx 20 \mathrm{~K}$ coexists with superconductivity in $t$-FeS [20]. There has also been evidence that a magnetic anomaly exists below $15 \mathrm{~K}$, while commensurate antiferromagnetic order exists below $116 \mathrm{~K}$ [17].

In this paper, we perform muon spin rotation experiments on a powder sample of $t$-FeS, in order to determine its magnetic and superconducting properties. Our results show that, while the superconducting properties of our samples match those in previous studies, the magnetic behavior is rather different, highlighting both the robustness of the superconducting state to magnetic disorder and the sensitivity of the magnetism to details of sample preparation.

\section{SAMPLE PREPARATION}

A sample of $t$-FeS was synthesized via a slightly modified literature procedure [10]. Elemental Fe powder (ALFA, $99.998 \%$ ) and anhydrous $\mathrm{Na}_{2} \mathrm{~S}$ (synthesized by the reaction of elemental sodium and sulfur in the correct ratio in liquid ammonia at $-50^{\circ} \mathrm{C}$ ) were weighed out in a 1:1 molar ratio and sealed inside a Teflon-lined steel autoclave after being solvated with deionized water $(10 \mathrm{ml})$. The reaction was heated at $120^{\circ} \mathrm{C}$ for 6 days before being furnace cooled to room temperature. The material was washed with deionized water and dried under vacuum. X-ray powder diffraction measurements showed the presence of elemental $\mathrm{Fe}$ within the sample. As such the isolated sample was reacted with a further 0.5 molar equivalents of anhydrous $\mathrm{Na}_{2} \mathrm{~S}$ in a Teflon-lined steel autoclave for 3 days at $130^{\circ} \mathrm{C}$ before being furnace cooled to room temperature. The dark gray powder was then washed with deionized water and dried under vacuum. Magnetometry measurements (using a Quantum Design SQUID magnetometer) confirmed that the sample was superconducting with a $T_{\mathrm{c}} \approx 4 \mathrm{~K}$ [see Fig. 1(a)].

\section{III. $\mu$ SR EXPERIMENTS}

$\mu$ SR experiments $[21,22]$ were performed using a $\mathrm{He}^{3}$ cryostat inserted in the MuSR spectrometer at the ISIS pulsed 

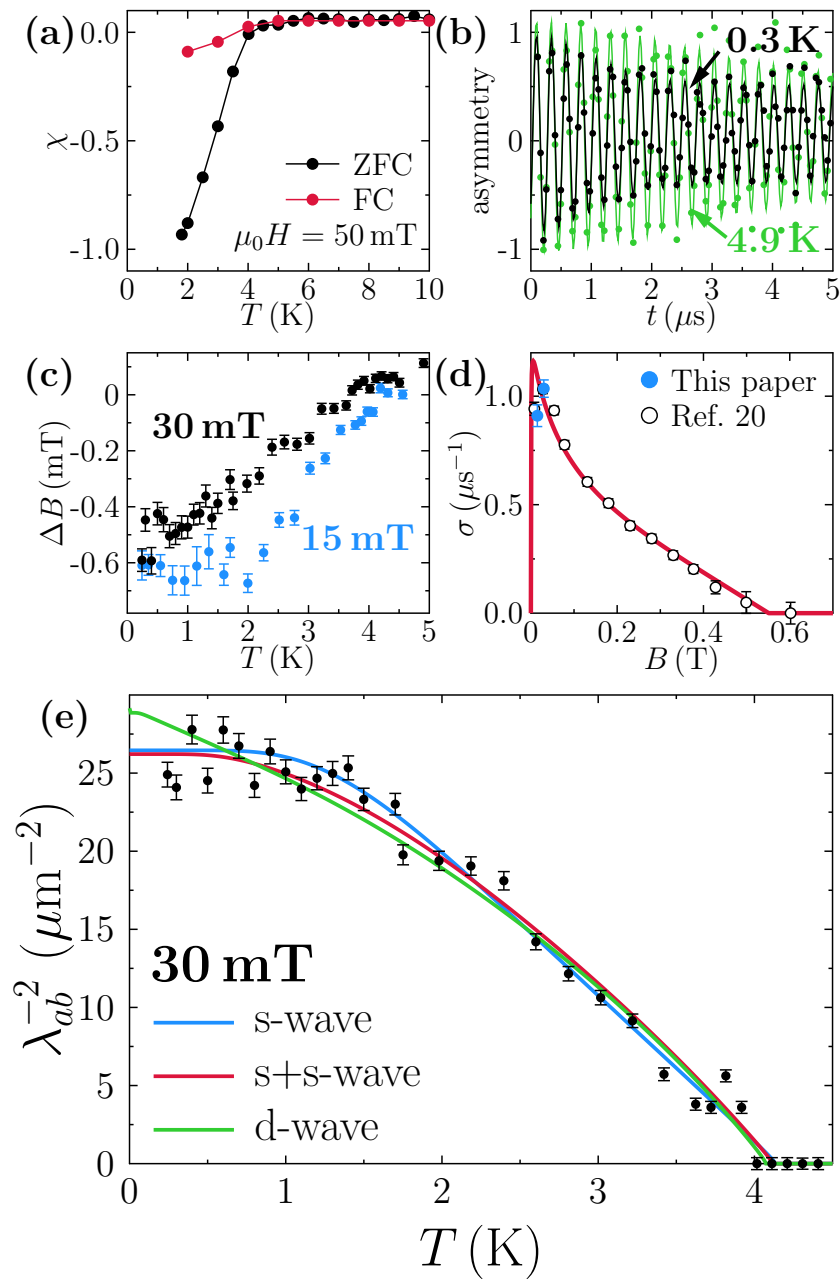

FIG. 1. (a) The field cooled (FC) and zero-field cooled (ZFC) magnetic susceptibility, $\chi=M / H$ where $M$ is the sample's magnetization, for $t$-FeS (given in dimensionless units) in a magnetic field of $\mu_{0} H=50 \mathrm{mT}$. (b) Sample TF $\mu$ SR spectra above and below $T_{\mathrm{c}}$ at an applied field of $30 \mathrm{mT}$, where the raw counts from one set of detectors has been plotted. (c) The shift in magnetic field $\Delta B=B_{\mathrm{SC}}-B_{\text {app }}$ due to the superconducting vortex lattice in applied fields of 15 and $30 \mathrm{mT}$. (d) Zero-temperature relaxation for our measurements, and those from Ref. [20], fitted to Eq. (2) with a low-field correction factor (black line). (e) The inverse square perpendicular penetration depth $\lambda_{a b}^{-2}$ at an applied field of $30 \mathrm{mT}$, calculated from $\mathrm{TF} \mu \mathrm{SR}$ spectra fitted with Eq. (1). Superfluid density functions for $s$-wave, $s+s$-wave, and $d$-wave superconductivity have been fitted as in Eq. (4).

muon facility (Rutherford Appleton Laboratory, UK) [23]. Initially, transverse-field (TF) measurements were performed in which a field was applied perpendicular to the initial direction of muon polarization to determine the nature of the superconductivity and the critical temperature. Next, zero-field (ZF) measurements were taken to determine which, if any, competing magnetic phases were present in the sample. For both sets of measurements, the sample was mounted on a hematite backing plate in order to remove the background signal which arises from the sample holder, causing a reduction in the oscillating (TF) and relaxing (ZF) amplitude in our data. All of the data were analyzed using WIMDA [24].

\section{A. TF measurements}

TF measurements above and below $T_{\mathrm{c}}$ were performed in magnetic fields, $B_{\text {app }}$, of 30 and $15 \mathrm{mT}$ between temperatures, $T$, of $0.24 \mathrm{~K}$ and $4.9 \mathrm{~K}$, and sample spectra are given in Fig. 1(b). There is a clear increase in relaxation below $T_{\mathrm{c}}$, caused by the onset of the superconducting vortex state, which produces an inhomogenous magnetic field distribution inside the sample [25]. The normalized data were fitted with the two-component function

$$
\begin{aligned}
A(t)= & A_{B} \cos \left(\gamma_{\mu} B_{\mathrm{app}} t+\phi\right) \exp \left[-\lambda_{\mathrm{TF}} t\right] \\
& +A_{\mathrm{SC}} \cos \left(\gamma_{\mu} B_{\mathrm{SC}} t+\phi\right) \exp \left[-(\sigma t)^{2} / 2\right],
\end{aligned}
$$

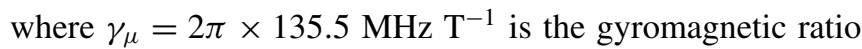
of the muon and $\phi$ is related to the detector geometry (the data were divided among eight groups of detectors with $\phi$ fitted for each group). The first term corresponds to muons that do not experience any superconductivity and precess in the external field. There exists a weak exponential relaxation $\left(\lambda_{\mathrm{TF}} \approx 0.03 \mu \mathrm{s}^{-1}\right)$ which arises from magnetism in the sample (see Sec. III B), but we cannot exclude that this term also accounts for a small contribution from muons implanted in the cryostat. The second term corresponds to the superconducting fraction of the sample, and the Gaussian broadening $\sigma(T)=$ $\sqrt{\sigma_{\mathrm{SC}}^{2}(T)+\sigma_{\text {nucl }}^{2}}$ contains contributions from the vortex lattice (which is temperature dependent) and the nuclear moments; we find $\sigma_{\text {nucl }}=0.368(3) \mu \mathrm{s}^{-1}$ and hence can deduce $\sigma_{\mathrm{SC}}$ from fitted values of $\sigma$. The best fit was found with $A_{\mathrm{B}}=0.56$ and $A_{\mathrm{SC}}=0.44$.

Figure 1(c) shows that there is a clear small negative shift in the average field due to the vortex lattice, $\Delta B=B_{\mathrm{SC}}-B_{\text {app }}$ caused by the distribution of fields of the vortex lattice (whose average field is slightly lower than the applied field), which decreases as $T_{\mathrm{c}}$ is approached and vortices disappear.

As the sample is anisotropic and polycrystalline, it can be assumed that the effective penetration depth $\lambda_{\text {eff }}$ is dominated by the in-plane penetration depth $\lambda_{a b}$ since $\lambda_{a b} \ll \lambda_{c}$, and so $\lambda_{\text {eff }}=3^{1 / 4} \lambda_{a b}$ [26]. Assuming $t$-FeS is a type II superconductor with an isotropic hexagonal Abrikosov vortex lattice in the $a-b$ plane that can described by Ginzburg-Landau theory, the relaxation due to the vortex lattice can be related to the penetration depth by the following approximation [27]:

$$
\sigma_{\mathrm{SC}}(T)=0.0993 \frac{\gamma_{\mu} \phi_{0}}{2 \pi}(1-b)\left[1+1.21(1-\sqrt{b})^{3}\right] \lambda_{a b}^{-2}(T),
$$

where $b=B_{\text {app }} / B_{\mathrm{c} 2}$ is the reduced field and $\phi_{0}=2.069 \times$ $10^{-15} \mathrm{~Wb}$ is the magnetic flux quantum. $\sigma_{\mathrm{SC}}$ is given in units of $\mu \mathrm{s}^{-1}$ and $\lambda_{a b}^{-2}$ in units of $\mu \mathrm{m}^{-2}$. This expansion holds to within $5 \%$ for $\kappa \geqslant 5$ and $0.25 / \kappa^{1.3}<b<1$, where the GinzburgLandau parameter of $t$ - $\mathrm{FeS}$ is $\kappa \approx 9$ [20].

The behavior of $\sigma_{\mathrm{SC}}$ can be extended to lower fields using an additional correction to Eq. (2) that takes account of the failure of the approximation $G \lambda \gg 1$ in the London formula $(\delta B)^{2}=\sum_{\mathbf{G} \neq 0} B_{\text {app }}^{2} /\left(1+G^{2} \lambda_{\text {eff }}^{2}\right)^{2}$, where $\mathbf{G}$ is the set of reciprocal lattice vectors [27]. The width of the field distribution is related to the relaxation by $\delta B=\sigma_{\mathrm{SC}} / \gamma_{\mu}$. Figure 1(d) shows the product of this correction factor (which $\approx 1$ for $B_{\text {app }} \gtrsim 0.25 B_{\mathrm{c} 2} / \kappa^{1.3}$ ) and Eq. (2). Our data only 
cover the low-field region, but are entirely consistent with those obtained in Ref. [20]. Fitting both data sets with this correction to Eq. (2) yields a value of the upper critical field of $B_{\mathrm{c} 2}=0.55(3) \mathrm{T}$ and a penetration depth of $\lambda_{a b}(0)=$ 204(3) $\mathrm{nm}$.

The parameter $\Delta B$ plotted in Fig. 1(c) is governed by the penetration depth: in $t$-FeS this is relatively large, resulting in the small $\Delta B$. The low-temperature measured $\Delta B$ values are close to the theoretical predictions: $\Delta B_{30 \mathrm{mT}} \approx-0.93 \mathrm{mT}$ and $\Delta B_{15 \mathrm{mT}} \approx-0.97 \mathrm{mT}$ as calculated using the approximation [27]

$$
\Delta B \approx-0.146 B_{\mathrm{c} 2} \frac{1-b}{\kappa^{2}-0.069} .
$$

The experimental values are slightly lower in magnitude than the theoretical values, and this can be explained by the Gaussian approximation made when fitting the vortex lattice field distribution in Eq. (1): in reality, the field distribution is not symmetric and is skewed towards lower fields, resulting in a larger shift [25].

Figure 1(e) shows $\lambda_{a b}^{-2}$ as a function of temperature at $30 \mathrm{mT}$ (very similar results were obtained for $15 \mathrm{mT}$ ). The data have been fitted with BCS single- and two-gap $s$-wave models and a single-gap $d$-wave model. The BCS model of the normalized superfluid density of a superconductor is given by [28]

$$
\begin{aligned}
\tilde{n}_{\mathrm{s}}(0) & =\frac{\lambda^{-2}(T)}{\lambda^{-2}(0)} \\
& =1+\frac{1}{\pi} \int_{0}^{2 \pi} \int_{\Delta(\phi, T)}^{\infty} \frac{\partial f}{\partial E} \frac{E d E d \phi}{\sqrt{E^{2}-\Delta^{2}(\phi, T)}},
\end{aligned}
$$

where $\Delta(\phi, T)$ is the superconducting gap function and $f=\left[1+\exp \left(E / k_{\mathrm{B}} T\right)\right]^{-1}$ is the Fermi function. The gap function can be approximated by $\Delta(\phi, T)=$ $\Delta(\phi) \tanh \left\{1.82\left[1.018\left(T_{\mathrm{c}} / T-1\right)\right]^{0.51}\right\}$, where the angular function $\Delta(\phi)=\Delta_{0}$ in the $s$-wave model and $\Delta(\phi)=$ $\Delta_{0} \cos (2 \phi)$ in the $d$-wave model [29], where $\Delta_{0}$ is a constant. The two-gap $s$-wave model is a simple superposition of two single-gap $s$ waves weighted by a factor of $w: \tilde{n}_{\mathrm{s}}(T)=$ $w \tilde{n}_{\mathrm{s}}^{(1)}(T)+(1-w) \tilde{n}_{\mathrm{s}}^{(2)}(T)$.

The fitting favors fully gapped behavior but cannot easily distinguish whether a single-gap or a two-gap $s$-wave model is more appropriate. The $s$-wave model with $\Delta_{0}=0.52(1) \mathrm{meV}$ gives the lowest overall $\chi^{2}$, and so this is taken as the model of best fit, although there is little difference between this and the $s+s$-wave model. This conclusion contrasts with some other studies of $t$-FeS, which have found evidence for nodes in the gap function [30,31]. However, a previous $\mu$ SR study has also reported fully gapped behavior, though the best fit was for two $s$-wave gaps [20].

Our extracted critical temperature $T_{\mathrm{c}}=4.13(3) \mathrm{K}$ at $30 \mathrm{mT}$ is in close agreement with previous studies on $t$-FeS $[10,17,20,31,32]$.

\section{B. ZF measurements}

Despite the remarkable similarity in the superconducting properties between our sample and that reported in Ref. [20], the magnetic properties have been found to be markedly different. Sample ZF $\mu$ SR spectra above and below $T_{\mathrm{c}}$ are

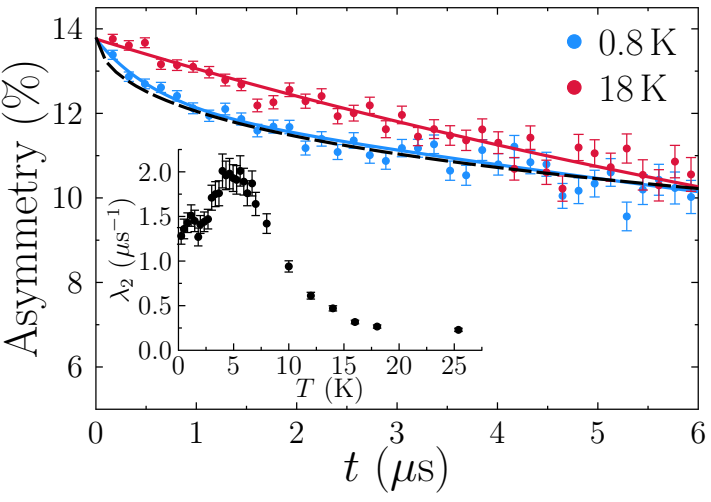

FIG. 2. Sample ZF $\mu$ SR spectra for $t$-FeS below and above $T_{\mathrm{c}}$. Two-component exponential fits, given by Eq. (5), are also plotted. The black dashed line shows a fit from a simulated dilute, dynamic spin system. The inset shows the temperature dependence of the relaxation rate of the fast-relaxing phase in Eq. (5).

given in Fig. 2. We observe a slight increase of relaxation as $T$ approaches $T_{\mathrm{c}}$, but this is not as drastic a change as reported in Ref. [20]. [Note that in Fig. 2 the initial $(t=0)$ asymmetry is only $\approx 14 \%$, lower than the maximum expected for data on this spectrometer, largely due to muons being absorbed by the hematite.] No oscillations were seen in the forward-backward asymmetry spectra, and there were also no discontinuous jumps in either the initial or the baseline asymmetry. This, combined with the absence of a KuboToyabe relaxation (ruling out effects from nuclear moments), hints that dynamic moments with no long-range order exist in the sample. This can be modeled by a phenomenological two-component exponential relaxation,

$$
A(t)=A_{1} \exp \left(-\lambda_{1} t\right)+A_{2} \exp \left(-\lambda_{2} t\right),
$$

which takes into account a slowly relaxing background with amplitude $A_{1}$ and relaxation rate $\lambda_{1}$, and a further signal with amplitude $A_{2}$ and a faster relaxation rate, $\lambda_{2}$. Exponential relaxation corresponds either to dynamic moments with a single correlation time within the resolution of the spectrometer and an unknown field distribution [33] or to a dilute distribution of static moments [34]. In this picture, the slower background relaxation (with constant $\lambda_{1} \approx 0.04 \mu \mathrm{s}^{-1}$ ) could arise due to the intrinsic magnetic moments of the iron in $t$-FeS (which is in contrast to behavior observed in FeSe [35]), whereas the faster relaxation could be due to a magnetic impurity phase. The inset in Fig. 2 shows the temperature dependence of the faster relaxation rate $\lambda_{2}$, measured between $0.24 \mathrm{~K}$ and $25.3 \mathrm{~K}$. As temperature decreases, the relaxation rate increases until $T \approx 5 \mathrm{~K}$ and drops slightly thereafter. This is characteristic for a magnetic phase that coexists and competes with a superconducting phase. As the peak coincides closely with the superconducting transition in $t$-FeS, it is likely that the onset of magnetism is coupled to the superconducting order parameter. It should be noted that at all measured temperatures $\lambda_{2}$ is large, but the relative amplitude $A_{2} /\left(A_{1}+A_{2}\right) \approx 15 \%$ is small, indicating a very low density of moments with a large distribution of stray fields.

We have carried out simulations to explore the effect of a system of dilute, dynamic spins which could arise 
from localized magnetic impurities within the FeS layers. We considered a random, inhomogeneous distribution of magnetic moments populating a lattice at low concentration and calculated the resulting dipolar field distribution at a test site, characterizing that distribution by the dipolar field width $\Delta / \gamma_{\mu}$. The muon relaxation rate is then given by $\int \rho(\Delta) e^{-\Delta^{2} t / v} d \Delta$, where $\rho(\Delta)$ is the probability density of $\Delta$ and $v$ is a characteristic fluctuation frequency. Our results are consistent with experimental observations if the concentration of such impurities is $\sim 1 \%$ and the fluctuation rate of these spins is $\approx 0.1-0.2 \mathrm{GHz}$ (this is shown in the black line plotted in Fig. 2), although it is possible to achieve good agreement with higher concentrations of impurity spins with correspondingly higher fluctuation rates. However, our x-ray diffraction data rule out any magnetic impurity phase at a higher concentration than the percent level, and lower concentrations have been excluded by the simulations (as they do not agree with the data). Simulations of static spin distributions also did not fit the data and so we conclude that the fluctuations of these dilute spins are important (the situation is reminiscent of effects observed in spin glasses [36]). The moments were found to be on the order of $\approx 1 \mu_{\mathrm{B}}$, which suggests the magnetic phase could arise due to atomic iron or iron-based impurities in the FeS layers, similar to those found in FeSe [4,37]. We think this more likely than the low-moment $\left(10^{-2}-10^{-3} \mu_{\mathrm{B}}\right)$ uniform magnetic phase of uncertain origin postulated by Ref. [20].

The $\mathrm{ZF}$ results obtained in this experiment are significantly different than those in previous $\mu \mathrm{SR}$ work on $t$-FeS. In Ref. [20], a magnetic transition was observed at $T \approx 20 \mathrm{~K}$, resulting in a sharp increase in the relaxation rate, peaking at $\lambda \approx 0.5 \mu \mathrm{s}^{-1}$ with the relative fraction of the magnetic phase $\approx 89 \%$. This is markedly different from the behavior observed in our sample and suggests that the magnetic properties of $t$-FeS are strongly sample dependent, a conclusion which is supported by Ref. [17].
The similarity of the superconducting properties demonstrates that the superconducting ground state is remarkably insensitive to these differences in magnetism. Moreover, this supports the conclusion that the gap is isotropic because a ground state with nodes might be expected to be far more susceptible to the presence of impurities, which may be the source of these sample-dependent differences.

\section{CONCLUSIONS}

In summary, we have carried out $\mathrm{TF}-$ and $\mathrm{ZF}-\mu \mathrm{SR}$ experiments on a sample of $t$-FeS to determine its superconducting and magnetic properties. It was found that the superconductivity in $t$-FeS agrees remarkably well with both previous experiments and theoretical predictions, and shows the robustness of the superconducting phase. The magnetic properties were found to be significantly different to those measured in other samples, which demonstrates the high sensitivity of the magnetic phase to small alterations in the synthesis process, and moreover it highlights the insensitivity of the superconducting phase to these changes. It is anticipated that the $T_{\mathrm{c}}$ of $t$-FeS could be enhanced with similar chemical modifications to FeSe. Our results support the notion that if $t$-FeS can be chemically modified using techniques similar to those that have led to an enhancement of $T_{\mathrm{c}}$ in $\mathrm{FeSe}$ intercalates, then the superconductivity of FeS layers may prove to be remarkably resilient.

\section{ACKNOWLEDGMENTS}

We thank EPSRC, UK (Grant No. EP/M020517/1) and the Leverhulme Trust (Grant No. RPG-2014-221) for funding support and P. Biswas for experimental support. F.K.K.K. thanks Lincoln College, Oxford, for a doctoral studentship. Part of this work was performed at the Science and Technology Facilities Council (STFC) ISIS Facility, Rutherford Appleton Laboratory. Data will be deposited on http://ora.ox.ac.uk.
[1] Y. Kamihara, H. Hiramatsu, M. Hirano, R. Kawamura, H. Yanagi, T. Kamiya, and H. Hosono, J. Am. Chem. Soc. 128, 10012 (2006).

[2] Y. Kamihara, T. Watanabe, M. Hirano, and H. Hosono, J. Am. Chem. Soc. 130, 3296 (2008).

[3] Z.-A. Ren, W. Lu, J. Yang, W. Yi, X.-L. Shen, Z.-C. Li, G.-C. Che, X.-L. Dong, L.-L. Sun, F. Zhou, and Z.-X. Zhao, Chin. Phys. Lett. 25, 2215 (2008).

[4] F.-C. Hsu, J.-Y. Luo, K.-W. Yeh, T.-K. Chen, T.-W. Huang, P. M. Wu, Y.-C. Lee, Y.-L. Huang, Y.-Y. Chu, D.-C. Yan, and M.-K. Wu, Proc. Natl. Acad. Sci. USA 105, 14262 (2008).

[5] S. Medvedev, T. M. McQueen, I. A. Troyan, T. Palasyuk, M. I. Eremets, R. J. Cava, S. Naghavi, F. Casper, V. Ksenofontov, G. Wortmann, and C. Felser, Nat. Mater. 8, 630 (2009).

[6] M. Burrard-Lucas, D. G. Free, S. J. Sedlmaier, J. D. Wright, S. J. Cassidy, Y. Hara, A. J. Corkett, T. Lancaster, P. J. Baker, S. J. Blundell, and S. J. Clarke, Nat. Mater. 12, 15 (2013).

[7] J.-F. Ge, Z.-L. Liu, C. Liu, C.-L. Gao, D. Qian, Q.-K. Xue, Y. Liu, and J.-F. Jia, Nat. Mater. 14, 285 (2015).
[8] H. Takahashi, A. Sugimoto, Y. Nambu, T. Yamauchi, Y. Hirata, T. Kawakami, M. Avdeev, K. Matsubayashi, F. Du, C. Kawashima, H. Soeda, S. Nakano, Y. Uwatoko, Y. Ueda, T. J. Sato, and K. Ohgushi, Nat. Mater. 14, 1008 (2015).

[9] A. P. Drozdov, M. I. Eremets, I. A. Troyan, V. Ksenofontov, and S. I. Shylin, Nature (London) 525, 73 (2015).

[10] X. Lai, H. Zhang, Y. Wang, X. Wang, X. Zhang, J. Lin, and F. Huang, J. Am. Chem. Soc. 137, 10148 (2015).

[11] U. Pachmayr, N. Fehn, and D. Johrendt, Chem. Commun. 52, 194 (2016).

[12] A. J. Devey, R. Grau-Crespo, and N. H. de Leeuw, J. Phys. Chem. C 112, 10960 (2008).

[13] C. K. H. Borg, X. Zhou, C. Eckberg, D. J. Campbell, S. R. Saha, J. Paglione, and E. E. Rodriguez, Phys. Rev. B 93, 094522 (2016).

[14] A. Subedi, L. Zhang, D. J. Singh, and M. H. Du, Phys. Rev. B 78, 134514 (2008)

[15] K. D. Kwon, K. Refson, S. Bone, R. Qiao, W.-L. Yang, Z. Liu, and G. Sposito, Phys. Rev. B 83, 064402 (2011).

[16] D. Vaughan and M. Ridout, J. Inorg. Nucl. Chem. 33, 741 (1971). 
[17] S. J. Kuhn, M. K. Kidder, W. M. Chance, C. dela Cruz, M. A. McGuire, D. S. Parker, L. Li, L. Debeer-Schmitt, J. Ermentrout, K. Littrell, M. R. Eskildsen, and A. S. Sefat, arXiv:1603.01598.

[18] D. Louca, K. Horigane, A. Llobet, R. Arita, S. Ji, N. Katayama, S. Konbu, K. Nakamura, T.-Y. Koo, P. Tong, and K. Yamada, Phys. Rev. B 81, 134524 (2010).

[19] C. H. Lee, K. Kihoua, A. Iyoa, H. Kitoa, P. M. Shirage, and H. Eisaki, Solid State Commun. 152, 644 (2012).

[20] S. Holenstein, U. Pachmayr, Z. Guguchia, S. Kamusella, R. Khasanov, A. Amato, C. Baines, H.-H. Klauss, E. Morenzoni, D. Johrendt, and H. Luetkens, Phys. Rev. B 93, 140506 (2016).

[21] S. J. Blundell, Contemp. Phys. 40, 175 (1999).

[22] A. Yaouanc and P. D. de Reotier, in Muon Spin Rotation, Relaxation, and Resonance: Applications to Condensed Matter (Oxford University, Oxford, 2011).

[23] P. J. C. King, R. de Renzi, S. P. Cottrell, A. D. Hillier, and S. F. J. Cox, Phys. Scr. 88, 068502 (2013).

[24] F. L. Pratt, Physica B (Amsterdam, Neth.) 289-290, 710 (2000).

[25] E. H. Brandt, Phys. Rev. B 37, 2349 (1988).

[26] V. I. Fesenko, V. N. Gorbunov, and V. P. Smilga, Physica C (Amsterdam, Neth.) 176, 551 (1991).

[27] E. H. Brandt, Phys. Rev. B 68, 054506 (2003).
[28] S. Chandrasekhar and D. Einzel, Ann. Phys. 505, 535 (1993).

[29] A. Carrington and F. Manzano, Physica C (Amsterdam, Neth.) 385, 205 (2003).

[30] T. P. Ying, X. F. Lai, X. C. Hong, Y. Xu, L. P. He, J. Zhang, M. X. Wang, Y. J. Yu, F. Q. Huang, and S. Y. Li, Phys. Rev. B 94, 100504(R) (2016).

[31] J. Xing, H. Lin, Y. Li, S. Li, X. Zhu, H. Yang, and H.H. Wen, Phys. Rev. B 93, 104520 (2016).

[32] J. Zhang, F. L. Liu, T. P. Ying, N. N. Li, Y. Xu, L. P. He, X. C. Hong, Y. J. Yu, M. X. Wang, W. G. Yang, and S. Y. Li, arXiv:1604.05254.

[33] R. Khasanov, H. Luetkens, A. Amato, H.-H. Klauss, Z.-A. Ren, J. Yang, W. Lu, and Z.-X. Zhao, Phys. Rev. B 78, 092506 (2008).

[34] R. E. Walstedt and L. R. Walker, Phys. Rev. B 9, 4857 (1974).

[35] R. Khasanov, K. Conder, E. Pomjakushina, A. Amato, C. Baines, Z. Bukowski, J. Karpinski, S. Katrych, H.-H. Klauss, H. Luetkens, A. Shengelaya, and N. D. Zhigadlo, Phys. Rev. B 78, 220510 (2008).

[36] Y. J. Uemura, T. Yamazaki, D. R. Harshman, M. Senba, and E. J. Ansaldo, Phys. Rev. B 31, 546 (1985).

[37] R. Hu, H. Lei, M. Abeykoon, E. S. Bozin, S. J. L. Billinge, J. B. Warren, T. Siegrist, and C. Petrovic, Phys. Rev. B 83, 224502 (2011). 